\title{
Editorial
}

\section{Recent Advances in RF Propagation Modeling for 5G Systems}

\author{
Mihajlo Stefanovic, ${ }^{1}$ Stefan R. Panic, ${ }^{2}$ Rausley A. A. de Souza, ${ }^{3}$ and Juan Reig ${ }^{4}$ \\ ${ }^{1}$ Faculty of Electrical Engineering, University of Nis, Nis, Serbia \\ ${ }^{2}$ Faculty of Natural Science and Mathematics, University of Pristina, Kosovska Mitrovica, Serbia \\ ${ }^{3}$ National Institute of Telecommunications (Inatel), Santa Rita do Sapucaí, MG, Brazil \\ ${ }^{4}$ Institute of Telecommunications and Multimedia Applications (iTEAM), Universitat Politècnica de Valencia, Valencia, Spain
}

Correspondence should be addressed to Stefan R. Panic; stefanpnc@yahoo.com

Received 30 July 2017; Accepted 30 July 2017; Published 15 October 2017

Copyright (C) 2017 Mihajlo Stefanovic et al. This is an open access article distributed under the Creative Commons Attribution License, which permits unrestricted use, distribution, and reproduction in any medium, provided the original work is properly cited.

Continuously increasing demand for higher data rates, larger network capacity, higher energy efficiency, and higher mobility has motivated research within fifth-generation $(5 \mathrm{G})$ communication systems modeling. 5G is generally agreed for a set of new requirements for wireless communications systems. These requirements will need to address several critical performance areas including cost constraints, traffic latency, reliability, security, availability, heterogeneous structure of networks, multicast/broadcast requirements, the requirement to serve a variety of different devices, and reduced energy consumption. Accurate 5G indoor and outdoor channel characterization and modeling are crucial for determining the system performance and thus for system and for $5 \mathrm{G}$ network realization. Namely, 5G radio frequency (RF) propagation is affected by various phenomena that more or less deteriorate the original transmitted signal arriving at the receiver (free-space propagation, object penetration, reflection, scattering, diffraction, and absorption caused by atmospheric gases, fog, and precipitation).

To generate reliable propagation models for $5 \mathrm{G}$ systems and further to determine standard performance measurements of $5 \mathrm{G}$ systems, corresponding path loss models must be built for link budget evaluation and signal strength prediction, with the inclusion of directional and beamforming antenna arrays and cochannel interference, while temporal dispersion caused by multipath propagation (impacting the timing, packet and frame sizes, and other air interface design parameters) should also be characterized. Therefore, general statistical models could not be sufficient in order to assess the performance of system and specific models related to real-world reference scenarios with fine classification of terms will be required.

For the development of new $5 \mathrm{G}$ systems to operate in millimeter bands, there is a need for accurate propagation modeling at these bands. Exploitation of unused millimeter wave (mmWave) band spectrum (spectrum between 6 and $300 \mathrm{GHz}$ ) is an efficient solution for meeting the standards for $5 \mathrm{G}$ networks enormous data demand growth explosion. Measurements provided at $38 \mathrm{GHz}$ (Base Station-to-Mobile Access Scenario [1] and Peer-to-Peer Scenario [2]), $60 \mathrm{GHz}$ (Peer-to-Peer Scenario and Vehicular Scenario [3]), and $73 \mathrm{GHz}$ [4] have clearly identified the existence of nonline-of-sight (NLOS) conditions. One of the most intensively used statistical models for characterizing the complex behavior and random nature of NLOS fading envelope is the Nakagami- $m$ distribution. In [5-7] for the purpose of modeling observed 5G system propagation properties, the Nakagami- $m$ parameter is directly computed from the measured data. Two most well-known procedures used for the estimation of the Nakagami- $m$ fading parameter, $m$, are (1) maximum likelihood (ML) estimation and (2) momentbased estimation. However, it is known that sample moments are often subjected to the effects of outliers (even a small portion of extreme values, outliers, can affect the Gaussian parameters, especially the higher order moments). Moreover, occurrence of outliers is especially problematic when higher order sample moments are used for estimation, since estimation inaccuracy arises in such cases. Providing the best 
moment-based estimator is still major issue that should be addressed.

Because of that, there is a need for developing a novel approach for NLOS channels parameter estimation based on performance measurements, which will enable us to estimate propagation parameters in real time and to avoid weaknesses of ML and moment-method estimation approaches.

Stochastic channel models for mmWave communications in both indoor and outdoor environments have been mostly characterized with a Rician distribution in line-of-sight (LOS) environments where a dominant path is present [8]. In [8] it has been shown that Ricean $K$-factor is ranging over a defined set of values for observed LOS and NLOS conditions in vertical-to-vertical $(\mathrm{V}-\mathrm{V})$ copolarized antenna scenario and corresponding set of values for observed LOS and NLOS conditions vertical-to-horizontal (V-H) crosspolarized antenna scenario. However, despite the fact shown in [8] that Rician distribution provides the best fit to the measurement data, results of [8] imply that conventional fading models fall short of accurate modeling of the random fluctuations of $5 \mathrm{G}$ wireless channel signal. In [9] it has been concluded that for accurate $5 \mathrm{G}$ systems channel modeling, proposed models should ensure that the channel LOS and NLOS conditions, the second-order statistics of the channel, and the channel realizations should change smoothly in the function of time, antenna position, and/or frequency. Therefore a need arises for novel characterization of propagation in LOS conditions, by observing Ricean $K$-factor as a random process.

In particular, the diversity of scenarios envisaged for the $5 \mathrm{G}$ applications at the mmWave band will certainly lead to a variety of propagation conditions. Currently, an enormous variety of waveforms are considered to be potential candidates for the $5 \mathrm{G}$ air interface. They include (i) single-carrier frequency division multiplexing (SC-FDMA), already used in 4G Long-Term Evolution (LTE) uplink, also called differently generalized discrete Fourier transforms- (GDFTs-) orthogonal frequency division multiplexing (OFDM) [10]; (ii) zerotail (ZT) or unique-word (UW) DFT-spread-OFDM [11, 12], ultra-wideband- (UW-) OFDM, generalized frequency division multiplexing (GFDM) [13], and cyclic prefix- (CP-) OFDM (already used in the 4G LTE downlink); (iii) resourceblock-filtered OFDM, filter-bank-multicarrier (FBMC), and universal filter multicarrier (UFMC). As it can be seen, the OFDM technique is omnipresent in the $5 \mathrm{G}$ waveform proposals. The OFDM technique shall certainly remain as the root framework for the new $5 \mathrm{G}$ waveform design, with some optimization to support the new $5 \mathrm{G}$ requirements $[14,15]$.

Therefore it is necessary to propose an efficient, simple, and general method to generate samples for general $5 \mathrm{G}$ channel and further to make use of this channel in order to assess the bit error rate performance of an OFDM system model.

Stochastic geometry has been a powerful technique to evaluate system performance in conventional cellular networks [16], which reveals the impacts of multiple system parameters such as base station density, transmit power, and path loss exponent on the performance parameters such as data rate or reliability. The key idea in [17] is to model random obstacles (e.g., buildings) as rectangles with random sizes and orientations whose centers form a Poisson point process (PPP) in 2-dimensional space. However, instead of one-hop communication between the macro cell base station (MBS) and a single small cell base station (SBS) cluster, the $5 \mathrm{G}$ cellular networks may have multiple SBS clusters, which require multihop transmissions to improve the cell coverage. Considering the distance-dependence of the blockage effects (i.e., the likelihood of a blockage event increases as the distance increases) at mmWave, multihop communication can be an effective solution to build mmWave wireless backhaul systems. In this context, motivated by the limitation in [18], the single-hop wireless backhaul system from [18] can be extended to a multihop scenario with multiple SBS clusters. Multiple points-to-multiple-points (MBMs-to-MBMs) links could be also studied, instead of [18] single-point-tomultiple-points (MBS-to-SBSs) links. Therefore, with different distance statistics from [18], the intercluster SBS-toSBS communication can benefit from higher order of spatial diversity compared to the MBS-to-SBS communication in [18].

From this point of view, the analysis of an optimal and suboptimal hop count to minimize the end-to-end outage performance between the MBS and the destination SBS cluster for a given end-to-end distance could be of interest, where the suboptimal hop count is based on only the per-hop outage performance.

Interference issues will become of crucial importance due to the coexistence of $5 \mathrm{G}$ devices, since a number of mmWave devices are expected to grow extensively in the near future [19]. In order to satisfy 5G quality-of-standard requirements and meet user mobility, due to the higher path loss at mmWave frequency range, multiple antenna arrays could be used in outdoor mmWave systems for providing an additional gain $[20,21]$. The increasing growth of $5 \mathrm{G}$ devices number will prompt the study of array pattern nulling techniques. The objectives of design of the antenna arrays are to achieve a minimum side lobe level (SLL) and a narrow first null beam width (FNBW). Methods used for the antenna array synthesis can be classified into two categories: deterministic and stochastic. The biggest advantages of using stochastic methods are their ability in dealing with large number of optimization parameters and avoiding getting stuck in local minimum.

An interesting idea for $5 \mathrm{G}$ mmWave antenna array synthesis could be based on genetic algorithm for the synthesis of linear array with nonuniform interelement spacing in order to obtain the optimal position of the elements in order to obtain the minimum side lobe level and nulls in desired directions.

The use of mmWave bands for next-generation wireless systems could offer ultra-wideband spectrum availability and increased channel capacity. All these benefits come at the expense of potentially higher system complexity particularly in terms of radio frequency (RF) front end and antenna design. However, the recent advancements around mmWave wireless systems development have produced cost-effective solutions that can be leveraged to overcome these challenges. $60 \mathrm{GHz}$ frequency band has its own standardized protocol, 
that is, the Wireless Gigabit Alliance (WiGig) standard which is equivalent to IEEE 802.11ad [22]. A promising study would be the link budget estimation, performed based on WiGig/IEEE 802.11ad standard-defined modulation and coding scheme (MCS) modes and $60 \mathrm{GHz}$ mmWave specific path loss and auxiliary attenuation factors. The considered systems parameters for this link budget estimation could be obtained from real-world hardware prototypes for nextgeneration mmWave mesh backhaul networks in industry.

Although multiple-input and multiple-output (MIMO) techniques have been widely employed in cellular and wireless local area network systems working at sub- $6 \mathrm{GHz}$ [23-25], the potential realizations of MIMO technique in mmWave band are not fully understood yet, considering the unique multipath propagation characteristics and the increased path loss over the lower frequency bands used in current 3G/4G wireless communication. Spatial multiplexing $(\mathrm{SM})$ and beamforming $(\mathrm{BF})$ are the two most commonly used approaches to realize a MIMO system. The multiplexing gain can be obtained by exploiting the spatial difference of the channel response in different transmit- $(\mathrm{Tx}-)$ receive $(\mathrm{Rx})$ element pair. On the other hand, in the mmWave band, the propagation loss is higher compared to the lower frequencies; thus the high-gain antenna arrays are expected to compensate the increased path loss. Several researches have conducted analyses on the performance of SM and BF in the mmWave communications. The feasibility of indoor mmWave MIMO has been investigated by ray-tracing based channel modeling, by virtual antenna array based channel measurement $[26,27]$, and also by a $2 \times 2$ microstrip array in an underground mine environment [28]. The performance of a hybrid transmission combining $\mathrm{BF}$ and $\mathrm{SM}$ in mmWave communication is also analyzed based on a ray-tracing method in both LOS and multipath environment [29].

It could be of interest to provide the measurementbased channel capacity comparison between SM and BF under realistic antenna arrays, with the same Tx power, the same array position, and the same propagation condition. In particular, a SM system could be analyzed and divided into 4 subarrays, each one of them consisting of 4 elements, corresponding to a $4 \times 4 \mathrm{MIMO}$ system, while in the $\mathrm{BF}$ system, the antenna array could be constructed by the whole 16 elements, which corresponds to a single-input and singleoutput (SISO) system but with a larger array gain than that of the SM system.

The introduction of MIMO and receiver diversity wireless devices provides large gains in the throughput performance. These gains are highly dependent on the performance of the receiving-antenna system and the receiving algorithm [30]. The devices can change the behavior of the antenna systems, for example, by using beamforming mechanisms, and also can adapt software algorithms to suit the environment they are currently used in. Wireless equipment manufacturers as well as network providers are pushing to have performance tests of the hand-held devices. Network providers expect to recommend the user equipment (UE) with the best performance to their customers; manufacturers wish to be able to compare the quality of their own UE to the one of the competitors. These comparisons should include the effect of the antenna systems, the analog frontends, digital receiving algorithms, and baseband processing. One of the methods proposed by $3 \mathrm{GPP}$, yet very promising one, is the decomposition method (DM). Over-the-air (OTA) throughput tests of wireless MIMO devices are an important tool for network operators and manufacturers. The UE is placed in an anechoic chamber and a random fading process is emulated by a base station emulator (BSE). The antenna characteristic of the UE is taken into account by sampling the sphere around the UE with the BSE test antenna at a large number of positions. For low-variance throughput results, long measurement intervals over many fading realizations are required, leading to long and expensive measurement periods in an anechoic chamber. Analyzing the possibilities of speeding up OTA testing through upgrading methods for DM analysis could be interesting task in performing throughput testing of wireless MIMO devices.

Attenuation by a human body and trees and penetration losses of material at the ITU proposed frequency bands [31], 24.25-27.5 and 37-40.5 GHz, are important issues for future $5 \mathrm{G}$ wireless access systems. In [31] the attenuation by a human body and trees and penetration loss of different materials with $1 \mathrm{GHz}$ bandwidth were measured with a time domain channel sounder at 26 and $39 \mathrm{GHz}$, respectively. As far as we know, there are no measurements and modeling work reported in open literature on human blockage, attenuation by trees, and penetration loss of different materials at 24.25-27.5 and $37-40.5 \mathrm{GHz}$ frequency bands. The prediction of attenuation by a human body and trees and the penetration losses in this work are important and necessary for future mmWave wireless communication systems deployment. By considering a human body as an infinite absorbing screen, two knife-edge (KE) models were used to predict the attenuation by a person in a frequency range from 4 to $10 \mathrm{GHz}$ in [32]. In addition to regarding a human body as an absorbing screen, a cylindrical model by uniform theory of diffraction (UTD) was also applied to predict human body attenuation. Measurements in $[33,34]$ were performed at $10 \mathrm{GHz}$ which showed a strong correlation between a human body and a perfect conducing cylinder. Previous works about penetration losses of material in mmWave bands were focused on $28 \mathrm{GHz}$ and $60 \mathrm{GHz}$. In [35], signals through a hollow plasterboard wall resulted in a penetration loss ranging between $5.4 \mathrm{~dB}$ and $8.1 \mathrm{~dB}$. In [36], the measured penetration losses are $2 \mathrm{~dB}, 9 \mathrm{~dB}$, and $35.5 \mathrm{~dB}$ at $60 \mathrm{GHz}$ through a glass door, a plasterboard wall with metallic studs, and a wall with a metalbacked blackboard, respectively. For this reason, it would be of interest to carry out measurements of the attenuation at 26 and $39 \mathrm{GHz}$ by a human body and trees as well as penetration losses for material with a person lateral crossing the transceiver connection line and to use KE and UTD methods to predict its attenuation. Also it would be of interest to measure the attenuation by willow trees at $26 \mathrm{GHz}$ and then to compare with ITU-R P-833-8 model and modify the model at $26 \mathrm{GHz}$. An interesting investigation would also be carrying out measurements of the penetration loss for different materials as well, for example, transparent glass with different thickness, frosted glass, and wood with plastic clad. 
$5 \mathrm{G}$ wireless communication networks are expected to fulfill the demand for higher data rates, lower latency, and/or massive connectivity of a growing number of users/devices exploiting a variety of wireless applications. This envisioned rapid increase in the use of wireless services would lead the wireless research community to start looking at new technologies to address problems related to the RF propagation modeling. This includes the development of models for new concepts such as massive MIMO systems to improve the spectral efficiency at the link and network layers and developing novel propagation models for characterizing communication in particular in the upper mmWave.

Generally, we need to continue to progress in our research for appropriate $5 \mathrm{G}$ radio propagation models, which can adequately and faithfully model mmWave communication properties much more than what has been done at the moment. The progress reported in this Special Edition is just a small step in achieving this goal in the future.

\section{Mihajlo Stefanovic Stefan R. Panic Rausley A. A. de Souza Juan Reig}

\section{References}

[1] T. S. Rappaport, E. Ben-Dor, J. N. Murdock, and Y. Qiao, "Proceeding of the $38 \mathrm{GHz}$ and $60 \mathrm{GHz}$ angle-dependent propagation for cellular \& peer-to-peer wireless communications," in Proceedings of the IEEE International Conference on Communications (ICC '12), pp. 4568-4573, Ottawa, ON, Canada, June 2012.

[2] T. S. Rappaport, F. Gutierrez, E. Ben-Dor, J. N. Murdock, Y. Qiao, and J. I. Tamir, "Broadband millimeter-wave propagation measurements and models using adaptive-beam antennas for outdoor Urban cellular communications," IEEE Transactions on Antennas and Propagation, vol. 61, no. 4, pp. 1850-1859, 2013.

[3] E. Ben-Dor, T. S. Rappaport, Y. Qiao, and S. J. Lauffenburger, "Millimeter-wave $60 \mathrm{GHz}$ outdoor and vehicle AOA propagation measurements using a broadband channel sounder," in Proceedings of the 54th Annual IEEE Global Telecommunications Conference: "Energizing Global Communications" (GLOBECOM '11), Kathmandu, Nepal, December 2011.

[4] G. R. Maccartney Jr. and T. S. Rappaport, " $73 \mathrm{GHz}$ millimeter wave propagation measurements for outdoor urban mobile and backhaul communications in New York City," in Proceedings of the 1st IEEE International Conference on Communications (ICC '14), pp. 4862-4867, Sydney, Australia, June 2014.

[5] S. K. Yoo, S. L. Cotton, R. W. Heath, and Y. J. Chun, "Measurements of the $60 \mathrm{GHz}$ UE to eNB Channel for Small Cell Deployments," IEEE Wireless Communications Letters, vol. 6, no. 2, pp. 178-181, 2017.

[6] D. Beauvarlet and K. L. Virga, "Measured characteristics of 30$\mathrm{GHz}$ indoor propagation channels with low-profile directional antennas," IEEE Antennas and Wireless Propagation Letters, vol. 1, pp. 87-90, 2002.

[7] J. Reig, M.-T. Martínez-Inglés, L. Rubio, V.-M. RodrigoPeñarrocha, and J. Molina-García-Pardo, "Fading evaluation in the $60 \mathrm{GHz}$ band in line-of-sight conditions," International Journal of Antennas and Propagation, vol. 2014, Article ID 984102, 12 pages, 2014.
[8] M. K. Samimi, G. R. Maccartney, S. Sun, and T. S. Rappaport, " $28 \mathrm{GHz}$ millimeter-wave ultrawideband small-scale fading models in wireless channels," in Proceedings of the 83rd IEEE Vehicular Technology Conference, VTC Spring 2016, May 2016.

[9] K. Haneda, L. Tan, Y. Zheng et al., "5G 3GPP-like channel models for outdoor urban microcellular and macrocellular environments," in Proceedings of the 2016 IEEE 83rd Vehicular Technology Conference (VTC Spring), pp. 1-7, Nanjing, China, May 2016.

[10] G. Berardinelli, K. I. Pedersen, T. B. Sørensen, and P. Mogensen, "Generalized DFT-Spread-OFDM as 5G Waveform," IEEE Communications Magazine, vol. 54, no. 11, pp. 99-105, 2016.

[11] G. Berardinelli, F. M. L. Tavares, T. B. Sørensen, P. Mogensen, and K. Pajukoski, "Zero-tail DFT-spread-OFDM signals," in Proceedings of the 2013 IEEE Globecom Workshops, GC Wkshps 2013, pp. 229-234, Atlanta, Ga, USA, December 2013.

[12] A. Sahin, R. Yang, M. Ghosh, and R. L. Olesen, "An improved unique word DFT-spread OFDM scheme for 5G systems," in Proceedings of the IEEE Globecom Workshops, GC Wkshps 2015, USA, December 2015.

[13] N. Michailow, M. Matthe, I. S. Gaspar et al., "Generalized frequency division multiplexing for 5 th generation cellular networks," IEEE Transactions on Communications, vol. 62, no. 9, pp. 3045-3061, 2014.

[14] B. Farhang-Boroujeny and H. Moradi, "OFDM Inspired Waveforms for 5G," IEEE Communications Surveys \& Tutorials, vol. 18, no. 4, pp. 2474-2492, 2016.

[15] X. Zhang, L. Chen, J. Qiu, and J. Abdoli, "On the Waveform for 5G," IEEE Communications Magazine, vol. 54, no. 11, pp. 74-80, 2016.

[16] T. Bai, R. Vaze, and R. W. Heath, "Analysis of blockage effects on urban cellular networks," IEEE Transactions on Wireless Communications, vol. 13, no. 9, pp. 5070-5083, 2014.

[17] A. Ghosh, N. Mangalvedhe, R. Ratasuk et al., "Heterogeneous cellular networks: from theory to practice," IEEE Communications Magazine, vol. 50, no. 6, pp. 54-64, 2012.

[18] H. Jung and I.-H. Lee, "Outage analysis of millimeter-wave wireless backhaul in the presence of blockage," IEEE Communications Letters, vol. 20, no. 11, pp. 2268-2271, 2016.

[19] S. Rangan, T. S. Rappaport, and E. Erkip, "Millimeter-wave cellular wireless networks: potentials and challenges," Proceedings of the IEEE, vol. 102, no. 3, pp. 366-385, 2014.

[20] J. Zhang, X. Ge, Q. Li, M. Guizani, and Y. Zhang, "5G millimeter-wave antenna array: design and challenges," IEEE Wireless Communications Magazine, pp. 2-8, 2016.

[21] F. Long and C. Zhang, Signal Processing for 5G: Algorithms and Implementations, Wiley-IEEE, 2016.

[22] T. S. Rappaport, J. N. Murdock, and F. Gutierrez, "State of the art in $60-\mathrm{GHz}$ integrated circuits and systems for wireless communications," Proceedings of the IEEE, vol. 99, no. 8, pp. 1390-1436, 2011.

[23] H. Sampath, S. Talwar, J. Tellado, V. Erceg, and A. Paulraj, "A fourth-generation MIMO-OFDM broadband wireless system: design, performance, and field trial results," IEEE Communications Magazine, vol. 40, no. 9, pp. 143-149, 2002.

[24] Q. H. Li, G. J. Li, W. Lee et al., "MIMO techniques in WiMAX and LTE: a feature overview," IEEE Communications Magazine, vol. 48, no. 5, pp. 86-92, 2010.

[25] J. Kim and I. Lee, "802.11 WLAN: history and new enabling MIMO techniques for next generation standards," IEEE Communications Magazine, vol. 53, no. 3, pp. 134-140, 2015. 
[26] M.-T. Martinez-Ingles, D. P. Gaillot, J. Pascual-Garcia, J.-M. Molina-Garcia-Pardo, M. Lienard, and J.-V. Rodríguez, "Deterministic and experimental indoor mmW channel modeling," IEEE Antennas and Wireless Propagation Letters, vol. 13, pp. 1047-1050, 2014.

[27] S. Ranvier, C. Icheln, and P. Vainikainen, "Measurement-based mutual information analysis of MIMO antenna selection in the 60-GHz band," IEEE Antennas and Wireless Propagation Letters, vol. 8, pp. 686-689, 2009.

[28] I. Ben Mabrouk, J. Hautcoeur, L. Talbi, M. Nedil, and K. Hettak, "Feasibility of a millimeter-wave MIMO System for short-range wireless communications in an underground gold mine," IEEE Transactions on Antennas and Propagation, vol. 61, no. 8, pp. 4296-4305, 2013.

[29] S. J. Lee and W. Y. Lee, "Capacity of multiple beamformed spatial stream transmission in millimetre-wave communication channels," IET Communications, vol. 7, no. 12, pp. 1263-1268, 2013.

[30] M. D. Foegelle, "Creating a complex multipath environment simulation in an anechoic chamber," Microwave Journal, vol. 53, no. 8, pp. 56-64, 2010.

[31] ITU, in Proceedings of the World Radio Communication Conference (WRC '15), Geneva, Switzerland, 2015, ITU 500-E.

[32] J. Kunisch and J. Pamp, "Ultra-wideband double vertical knifeedge model for obstruction of a ray by a person," in Proceedings of the IEEE International Conference on Ultra-Wideband (ICUWB '08), pp. 17-20, Hannover, Germany, September 2008.

[33] M. Ghaddar, L. Talbi, and T. A. Denidni, "Human body modelling for prediction of effect of people on indoor propagation channel," IEEE Electronics Letters, vol. 40, no. 25, pp. 1592-1594, 2004.

[34] M. Ghaddar, L. Talbi, T. A. Denidni, and A. Sebak, "A conducting cylinder for modeling human body presence in indoor propagation channel," IEEE Transactions on Antennas and Propagation, vol. 55, no. 11, pp. 3099-3103, 2007.

[35] E. J. Violette, R. H. Espeland, R. O. DeBolt, and F. Schwering, "Millimeter-wave propagation at street level in an urban environment," IEEE Transactions on Geoscience and Remote Sensing, vol. 26, no. 3, pp. 368-380, 1988.

[36] K. Sato, T. Manabe, T. Ihara et al., "Measurements of reflection and transmission characteristics of interior structures of office building in the 60-GHz band," IEEE Transactions on Antennas and Propagation, vol. 45, no. 12, pp. 1783-1792, 1997. 


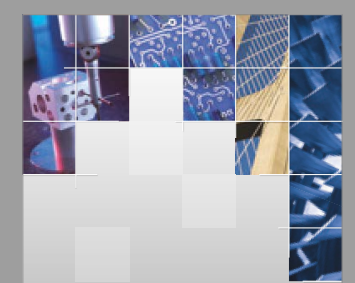

\section{Enfincering}
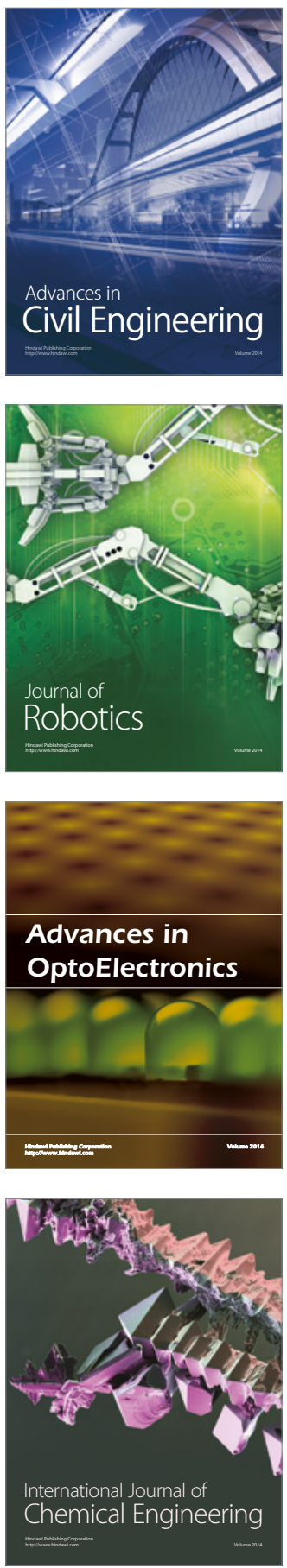

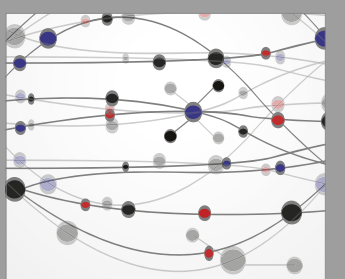

The Scientific World Journal

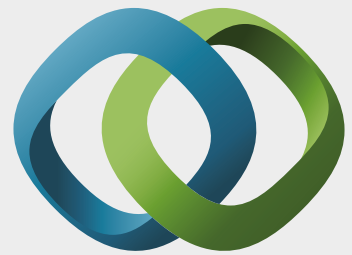

\section{Hindawi}

Submit your manuscripts at

https://www.hindawi.com
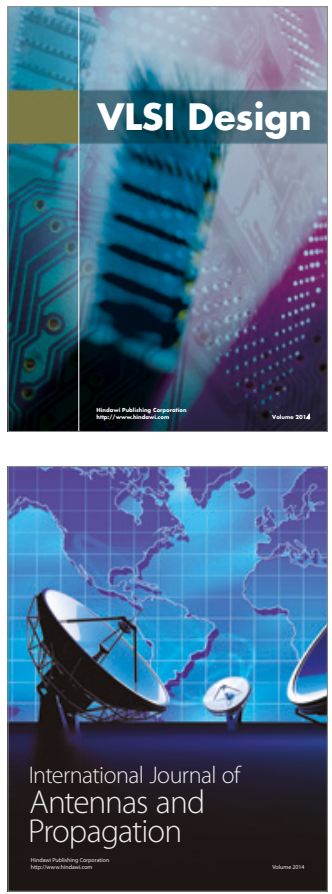

\section{Rotating}

Machinery
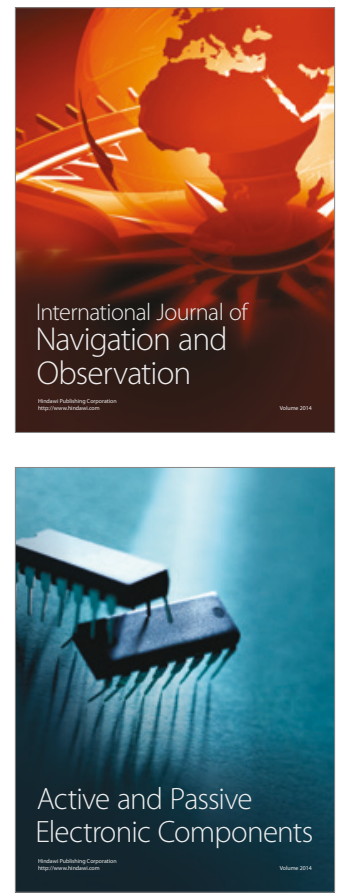
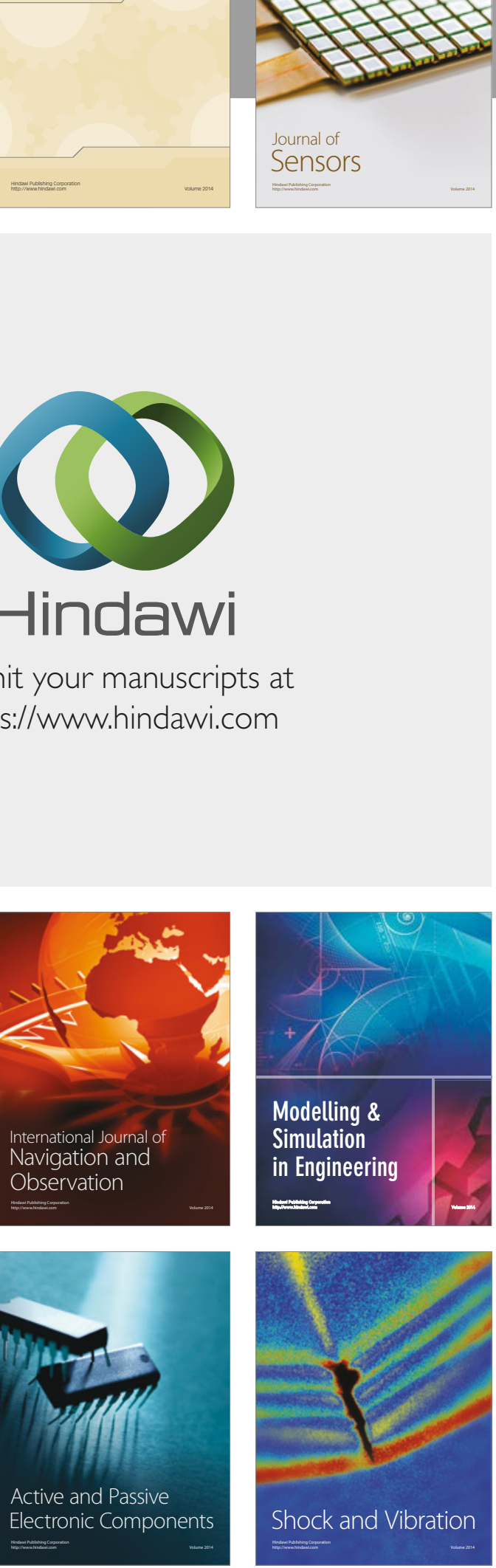
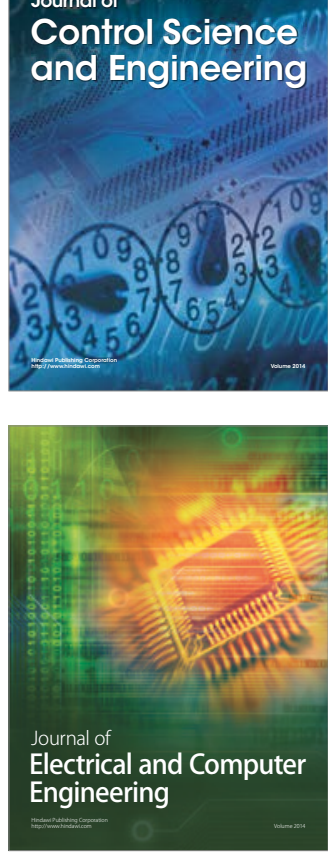

Distributed

Journal of

Control Science

and Engineering
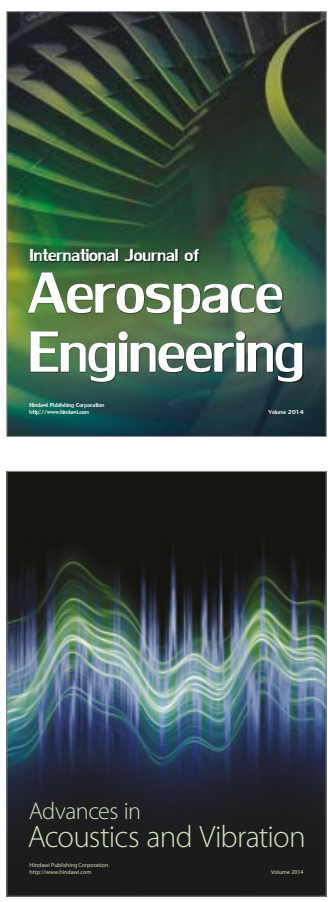

Sensor Networks 